\title{
Ungfågelspridning och höstflyttning hos svart rödstjärt Phoenicurus ochruros i södra Sverige
}

\author{
Dispersal of yearlings and autumn migration of Black Redstart Phoenicurus \\ ochruros in southern Sweden
}

REINO ANDERSSON

Abstract

The number of ringed Black Redstarts at Ottenby, Falsterbo and Nidingen bird observatories showed two distinct peaks after the breeding season. During the first peak in July-August, with 63, 67 and 89 percent of the total catch, the proportions of yearlings were 92, 100 and 96 percent, respectively, and most of them had not yet begun their postjuvenile moult and showed relatively low weights. During the second peak in October-November, all yearlings had completed this moult and carried more fat. Black Redstarts have a delayed postjuvenile moult, a unique strategy among European thrushes. This allows for wide dispersal during late summer. Several ringed
Belgian and German yearlings have moved up to $290 \mathrm{~km}$ northward during this time. This paper confirms, using ringing data and observations from a population study in western Sweden, that summer dispersal is well separated from autumn migration. I speculate that some of the birds of the July-August peak could have their origin in southern breeding populations, for example Denmark, where a major population increase has occurred in recent decades.

Reino Andersson, Rödaledstigen 6, S-432 53 Varberg, Sweden.E-mail: reino.andersson@ciaoip.se

\section{Inledning}

Svarta rödstjärten uppvisar årligen en kulmination av ringmärkta fåglar i juli-augusti vid fågelstationerna Ottenby, Falsterbo och Nidingen. Det rör sig främst om årsungar i juvenil dräkt, som efter flyggperioden har lämnat sina födelseplatser. Med juvenil avses här den första egentliga fjäderdräkten (Figur 1). Denna ersätts av adulta kroppsfjädrar i en partiell postjuvenil ruggning före höstflyttningen (Svensson 1992, Jenni \& Winkler 1994). Från Mellaneuropa hänvisar Biber (1973) till studier i de schweiziska Alperna, där en markant sträcktopp av huvudsakligen juvenila årsungar noterades i skiftet juli-augusti. Zink (1981) och Glutz \& Bauer (1988) anger flera fall där unga belgiska och tyskmärkta svarta rödstjärtar vid denna tidpunkt förflyttat sig även i nordlig riktning, som längst en sträcka på 29 mil.

Svarta rödstjärtens årsungar har en unik ruggningsstrategi jämfört med andra europeiska trastfåglar. Arter som rödstjärt och rödhake påbörjar normalt sin postjuvenila ruggning i födelseområdet endast några veckor efter att de blivit flygga (Glutz
\& Bauer 1988, Landmann 1996, Waldenström \& Bengtsson 2001). Svarta rödstjärtens ungfåglar startar däremot sin partiella kroppsruggning först då de uppnått en medelålder av 87 dagar (Berthold 1983, 1985, Glutz \& Bauer 1988, Landmann 1996). Detta ger utrymme för en ungfågelspridning under sommaren, vilket leder dem till nya platser där de genomför den partiella ruggningen. Det är

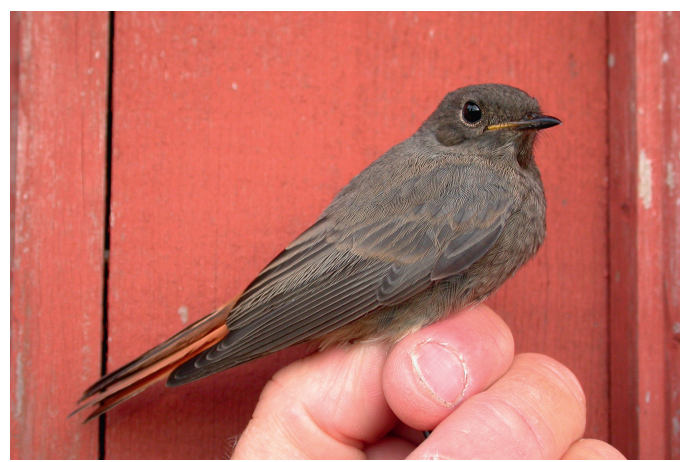

Figur 1. Svart rödstjärt i juvenil dräkt fotograferad på Nidingen 9 juli 2006. Foto: Uno Unger Black Redstart in typical juvenile plumage 9 july 2006. 
också känt att årsungar av blåhake kan påbörja sin flyttning innan de ruggat färdigt (Lindström $\mathrm{m}$. fl. 1985, Lindström \& Lind 2001).

Tidiga ungfågelrörelser bland tättingar är dåligt utforskade (Goodbody 1952, Baker 1978, 1993, Greenwood \& Harvey 1982, Boddy 1983, Norman 1991, Paradis m.fl. 1998, Raine m.fl. 2006). I Sverige har ämnet endast berörts kortfattat för svart rödstjärt (Knutsson 1981, Andersson 1986, 2001). Denna uppsats avser att utifrån ringmärkningsdata, vikt- och ruggningsuppgifter, ge en utförligare bild av ungfågelspridningens förlopp i förhållande till höstflyttningen. Det är särskilt motiverat då dessa spridningsrörelser ibland har uppfattats som en tidig inledning av höststräcket. Att sommarfynden är väl åtskilda från den egentliga höstflyttningen finns dock tidigare belagt från mellersta Europa (Menzel 1983, Glutz \& Bauer 1988). Berthold (1983, 1985) anger att flyttningsoron och det observerade huvudsträcket hos årsungar infaller betydligt senare i oktober-december, i medeltal två veckor efter avslutad ruggning. I samband med den ökade flyttningsoron, noterades dessutom en viktökning hos försöksfåglarna. Även adulta fåglar lämnar normalt sina häckningsplatser i oktober-november, efter en utpräglad sångperiod i september-oktober (Cramp 1988, Glutz \& Bauer 1988, Andersson 2001). En viss rörlighet tycks dock även förekomma hos äldre hannar, framför allt om de förblivit oparade. Ungfågelspridning bland tättingar i Sverige har därutöver beskrivits för bl.a. talgoxe, rörsångare, sävsångare och trastsångare (Ehrenroth 1976, Johansson 1980, Bensch \& Nielsen 1995, Stervander 1999). Bland icke-tättingar är ungfågelspridning ett mer välkänt begrepp och ofta uppges de tidiga sträckrörelserna ha en nordlig riktning (Otterlind 1954, Nielsen 1977).

\section{Material och metoder}

I materialet ingår ringmärkningsdata, vikt- och ruggningsuppgifter från Ottenby 1980-2011 samt Falsterbo och Nidingen 1980-2012. Den aktuella tidsperioden omfattar 20 juni-30 november. På Nidingen har fångsten varit kontinuerlig över säsongen, däremot inte standardiserad. Vid Ottenby har fångsten skett högst spontant fram till 25 juli, då standardiserad ringmärkning påbörjats. Vid Falsterbo har ringmärkningen, som varit standardiserad, startat först den 21 juli. Viktuppgifter presenteras här för att visa på en skillnad mellan sträcktoppen under sommaren och den egentliga höstflyttningen i oktober-november (resultaten har testats med t-test). Syftet med ruggningsuppgifterna är att bekräfta att ungfågelspridningen huvudsakligen sker i juvenil dräkt före ruggningsperioden och höstflyttningen. Klassningen av den postjuvenila ruggningen har följt den så kallade pullskalan, en poängsättning av olika ruggningsstadier, från helt juvenil dräkt utan inledd ruggning till helt färdigruggad första vinterdräkt (Lindström m.fl. 1985, Bensch \& Lindström 1992). Klassningen som redovisas i denna uppsats har dock anpassats efter de olika metoder som använts på fågelstationerna.

Dessutom har data medtagits från en tidigare populationsstudie med färgringmärkta individer i Västsverige, främst Göteborg, Varberg och Falkenberg (Andersson 1987, 1990, 1995, 2001). De berör rörelser efter häckningsperioden hos 19 av 188 ringmärkta boungar, 34 av 105 revirhävdande hannar och 8 av 20 honor ringmärkta samma vår. Detta för att ge en kompletterande bild av årsungarnas spridningsmönster, men också av adulternas eventuella rörelser inför höstflyttningen. Studieområdena som finns beskrivna i refererade uppsatser, besöktes i genomsnitt varannan dag under juli-augusti och var femte dag september-november 1983-1997. Fyra år (1988, 1990, 1993, 1994) gjordes dock färre besök under hösten med kontroller i genomsnitt var tolfte dag. Under dessa besök genomsöktes områdena efter så många fåglar som möjligt för att göra avläsningar av färgringar. Om inga fåglar sågs på tidigare besatta revir, spelades sång upp från bandspelare i högst två minuter, för att effektivisera upptäckten av färgringmärkta individer. På platser där fåglar iakttogs inom fem minuter, undveks däremot banduppspelning för minimera påverkan på deras beteenden.

\section{Resultat}

Antalet ringmärkta svarta rödstjärtar vid fågelstationerna hade två tydliga toppar under höstsäsongen (Figur 2). Den totala andelen årsungar uppgick till 86, 98 respektive $96 \%$ (Tabell 1). Under den tidiga sträcktoppen i juli-augusti var andelen något högre $(92,100$ respektive $96 \%)$. Vid Ottenby och Falsterbo ringmärks då $63 \%$ respektive $67 \%$ av den totala höstfångsten, medan motsvarande siffra för Nidingen uppgår till 89\%. I september märks sammantaget ytterst få fåglar, eftersom denna månad sammanfaller med årsungarnas ruggningsperiod. Vid Ottenby och Falsterbo făngades flera individer samma dag vid vardera 20 tillfällen, som mest fyra exemplar på respektive station. Vid Nidingen 


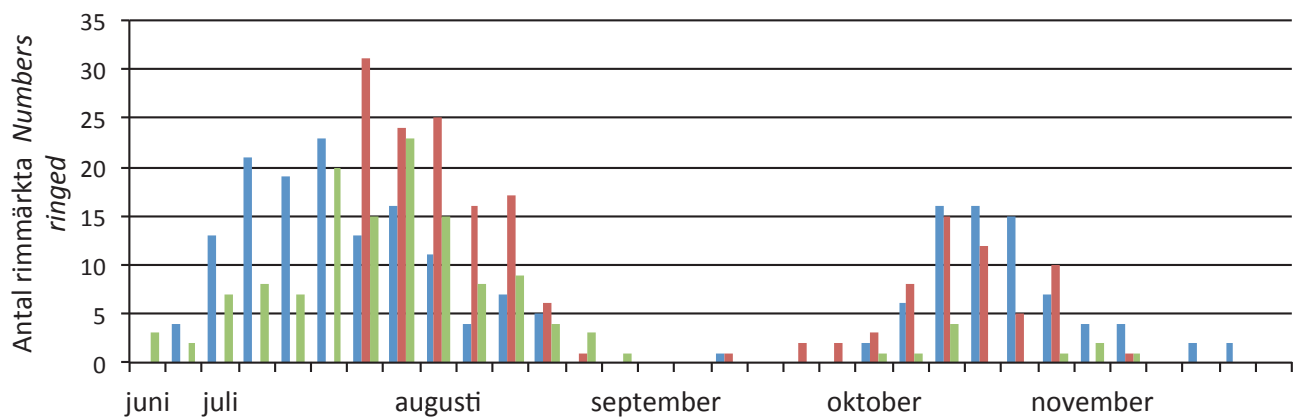

Figur 2. Antal ringmärkta svarta rödstjärtar per femdagarsperiod vid Ottenby, Falsterbo och Nidingen höstsäsongerna 19802012 (Ottenby t.o.m. 2011).

Number of ringed Black Redstarts per five day period at Ottenby, Falsterbo and Nidingen bird observatories during the autumn seasons 1980-2012 (Ottenby through 2011).

fångades som mest tre exemplar samma dag vid nio tillfällen.

Vid Ottenby registrerades påbörjad ruggning hos årsungar under juli-augusti i större utsträckning än vid Falsterbo och Nidingen (Tabell 2). Om man däremot slår samman de två första stadierna $(0,1)$, som representerar en huvudsakligen juvenil dräkt, uppgår procentandelen till 80,81 respektive $93 \%$. De årsungar som fångas senare på hösten har alla slutfört sin ruggning. Svarta rödstjärtar fångade under perioden juni-augusti var generellt lättare än de som fångades i oktober-november. Detta mönster var likadant vid samtliga tre fågelstationer. Medelvikten för Ottenbyfåglarna fångade $\mathrm{i}$ juni-augusti uppgick till 15,3 gram $(\mathrm{n}=106)$ och oktober-november 16,3 gram $(n=62, F=9,47, P<0,01)$. Vid

Tabell 1. Antal ringmärkta svarta rödstjärtar per månad vid Ottenby, Falsterbo och Nidingen höstsäsongerna 1980-2012 (Ottenby t.o.m. 2011).

Number of ringed Black Redstarts per month at Ottenby, Falsterbo and Nidingen bird observatories during the autumn seasons 1980-2012 (Ottenby through 2011).

\begin{tabular}{lrrrrrr}
\hline & \multicolumn{2}{c}{$\begin{array}{c}\text { Ottenby } \\
\text { Adulta }\end{array}$} & $\begin{array}{c}\text { Falsterbo } \\
\text { Adulta Årsungar }\end{array}$ & $\begin{array}{c}\text { Nidingen } \\
\text { Adulta }\end{array}$ \\
& Adults & Yearlings & Adults & Yearlings & Adults & Yearlings \\
\hline Juni & 0 & 4 & 0 & 0 & 1 & 4 \\
Juli & 10 & 95 & 0 & 55 & 4 & 76 \\
Augusti & 1 & 26 & 0 & 65 & 1 & 39 \\
September & 0 & 1 & 1 & 4 & 0 & 0 \\
Oktober & 17 & 45 & 2 & 51 & 0 & 7 \\
November & 2 & 10 & 0 & 1 & 0 & 3 \\
Total & 30 & 181 & 3 & 176 & 6 & 129 \\
\hline
\end{tabular}

Tabell 2. Antal ringmärkta årsungar av svart rödstjärt i olika ruggningsstadier* under juli-augusti vid Ottenby, Falsterbo och Nidingen höstsäsongerna 19802012 (Ottenby t.o.m. 2011). Ruggningspoängen har anpassats efter olika klassningsmetoder.

Number of ringed yearlings of Black Redstart in different moult stages* during July-August at Ottenby, Falsterbo and Nidingen bird observatories during the autumn seasons 1980-2012 (Ottenby through 2011). The moult score was adapted to different classification methods.

\begin{tabular}{lrrrrrr}
\hline Ruggningspoäng & \multicolumn{2}{c}{ Ottenby } & \multicolumn{2}{c}{ Falsterbo } & \multicolumn{2}{c}{ Nidingen } \\
Moult score & $\mathrm{n}$ & $\%$ & \multicolumn{1}{c}{$\mathrm{n}$} & $\%$ & $\mathrm{n}$ & $\%$ \\
\hline 0 & 2 & 4 & 60 & 60 & 59 & 86 \\
1 & 41 & 76 & 21 & 21 & 5 & 7 \\
2 & 5 & 9 & 14 & 14 & 5 & 7 \\
3 & 6 & 11 & 5 & 5 & 0 & 0 \\
\hline
\end{tabular}

*Ruggningsstadier Moult scores

0 = Ruggningen ej påbörjad. No moult occuring.

1 = Ruggar fjädrar på strupe och flanker men strupfjädrarna har ännu inte brutit ut ur spolarna (mindre än 10\% växande/ nya fjädrar). Moulting feathers on throat and sides of breast but throat feathers still in pin $(<10 \%$ growing/new feathers). 2 = Ruggar fjädrar på strupe och flanker (tre ruggningscentra). Strupfjädrarna har brutit ut ur spolarna (10-50\% växande/nya fjädrar). Moulting feathers on throat and sides of breast (three moulting areas). The throat feathers have now emerged from the sheaths (10-50\% growing/new feathers). $3=$ De tre ruggningscentra har vuxit ihop. Det går att följa ett sammanhängande stråk med nya fjädrar från strupen ner längs vardera kroppssidan (50-90\% växande/nya fjädrar). The three moulting areas have merged. There is an uninterrupted band of new feathers from the throat along each side of the breast and flanks (50-90\% growing/new feathers). 
Falsterbo var motsvarande siffror 15,0 $(\mathrm{n}=106)$ och $15,5(\mathrm{n}=45, \mathrm{~F}=12,95, \mathrm{P}<0,001)$. Medelvikten på Nidingen uppgick till 15,0 $(\mathrm{n}=122)$ respektive 16,5 $(\mathrm{n}=10, \mathrm{~F}=20,83, \mathrm{P}<0,001)$.

Av 188 färgringmärkta boungar i Västsverige påträffades 19 under höstsäsongen. Två var stationära 29 respektive 68 dygn, medan sjutton av dem iakttogs endast ett dygn på samma plats. Dessa ungar rörde sig till nya lokaler och avlästes som längst 7000 meter bort. Av 105 färgringmärkta, revirhävdande hannar i Västsverige iakttogs 34 under hösten. Nitton av dem stannade endast 1-5 dagar på samma plats. Sju av dessa påträffades senare 500-2000 meter bort, medan vidare observationer saknas. Åtta av de 20 honor som färgringmärktes under häckningssäsongen observerades under hösten. Samtliga var dock mycket stationära och rörelser registrerades som längst upp till 800 meter.

\section{Diskussion}

Ringmärkningsmaterialet från Ottenby, Falsterbo och Nidingen bekräftar att majoriteten av sommarfynden i juli-augusti utgörs av årsungar som inte har genomfört eller endast påbörjat sin postjuvenila ruggning. Detta indikerar tillsammans med lägre erhållna vikter, att denna sträcktopp inte utgörs av några egentliga flyttningsrörelser. Att årsungarna vid denna tidpunkt uppvisar en stor flexibilitet och rörlighet styrks, utöver individomsättningen på de olika stationerna, även av de färgringmärkta boungarnas uppträdande i Västsverige. I ett flertal fall ringmärktes på fågelstationerna flera individer under en och samma dag. Åtta fall från Västsverige ger belägg för att kullsyskon uppträtt tillsammans under sina rörelser mellan olika lokaler (Andersson 1990, 2001, egna opublicerade data). Detta skulle möjligen kunna innebära att ungfågelspridningen i vissa fall sker kollektivt. Att det i första hand rör sig om ungar från förstakullar kan uttolkas redan av Nesenhöner (1956), som anger att andrakullar vanligtvis stannar kvar längre $\mathrm{i}$ födelseområdet. Detsamma har noterats i Västsvenska häckningsområden, där andrakullar ofta varit kvar i sina födelseområden fram till bortflyttningen i oktober (Andersson 1990, 2001). Även en del adulta hannar visade på en förvånansvärt stor rörlighet under hösten, till skillnad från de äldre honorna som föreföll vara stationära på häckningsplatserna.

Varje höst sker ett markant tillskott av okända individer i de västsvenska häckningsområdena, vilket sannolikt härrör från en omfattande ungfågelspridning. En mindre del av påspädningen utgörs även av adulta individer, vilket antyds av vissa hannars dokumenterade rörlighet samt att majoriteten försvann från de västsvenska häckningsområdena redan under sommaren (Andersson 2001). Många av dem genomför antagligen längre förflyttningar och undgår därmed vidare upptäckt under hösten. Ett exempel på detta är en hanne som hävdade revir under tre veckor vid Ljunghusen i Skåne och som sjutton dagar senare kontrollerades vid Sundre på Gotland, cirka 35 mil i nordostlig riktning (Anonym 1981). Det är inte helt givet att fåglar norrifrån har störst inverkan på det okända individtillskottet. Årsungar kan även som tidigare konstaterats röra sig norrut (Zink 1981). Kanske utgörs istället majoriteten av individtillskottet $\mathrm{i}$ de västsvenska häckningsområdena, liksom uppträdandet på Nidingen och Falsterbo under sommaren, av svarta rödstjärtar från Sydsverige eller Danmark. Detta skulle delvis kunna förklara varför inga kontroller av kända västsvenska fåglar har gjorts på Nidingen, trots att cirka 20\% av Göteborgs årsungar periodvis varit färgringmärkta (egna opublicerade data). Inte heller ungar från häckningsområdena $\mathrm{i}$ mellersta Halland, där en ännu högre andel av årsungarna varit färgringmärkta, har resulterat i några kontroller varken på Nidingen eller i Falsterbo. I Danmark har en kraftig populationsökning ägt rum under senare decennier (Svensson m.fl. 1999, Danmarks Fugle, Dansk Ornitologisk Forening: www. dofbasen.dk/ART/). Samtidigt har den västsvenska populationen genomgått en drastisk minskning (Ottosson m.fl. 2012, egna opublicerade data). Man skulle också kunna tänka sig att Ottenbys ringmärkningssiffror kan ha en del av sitt ursprung i sydligare populationer. Hur långt årsungarna kan förflytta sig under denna årstid är ännu oklart. Det har dock framhållits att längre ungfågelrörelser kan spela en viktig roll för framtida revir- och häckningsetableringar (Baker 1993). Flera nordliga återfynd av Västsvenska årsungar föreligger under påföljande vår exempelvis i södra Norge, där en hona konstaterades häcka 25 mil NV födelseplatsen (Steel 1993). Det är inte otänkbart att den rört sig dit redan under sensommaren och genomfört sin första ruggning där.

Denna uppsats speglar främst spridningsförloppet hos årsungar under sommarmånaderna och den senare höstflyttningen hos svarta rödstjärten i södra Sverige. Mycket återstår dock att utforska när det gäller de enskilda individernas uppträdande under dessa spridningsrörelser. Hur deras detaljerade livsmönster ser ut är fortfarande höljt i dunkel, liksom strövtågens omfattning och ursprung. Utökade och riktade ringmärknings- 
insatser med fler återfynd och kontroller, skulle möjligen kunna avfärda eller bekräfta några av ovanstående spekulationer.

\section{Tack}

Ett stort tack riktas till Ottenby, Falsterbo och Nidingens fågelstationer som välvilligt ställt sina ringmärkningsmaterial till förfogande. Tack också till Magnus Hellström, Uno Unger, Bo Nielsen, Anders Nothagen, Pär Sandberg och Kåre Ström för granskning av uppsatsen eller intressanta diskussioner. Detta är meddelande nr. 272 från Ottenby fågelstation.

\section{Referenser}

Andersson, R. 1986. Ungfågelsträcket av svart rödstjärt på Nidingen 1980-1985. Fåglar på Västkusten 20: 1-4.

Andersson, R. 1987. Revirtäthet och populationsstruktur hos ett västsvenskt bestånd av svart rödstjärt Phoenicurus ochruros. Vår Fågelvärld 46: 256-269.

Andersson, R. 1990. Svarta rödstjärtens Phoenicurus ochruros häckningsbiologi i Västsverige. Vår Fågelvärld 49: 201-210.

Andersson, R. 1995. Hannars etableringsmönster, revirkvalitet samt flytande bestånd i en randpopulation av svart rödstjärt Phoenicurus ochruros. Ornis Svecica 5: 143-159.

Andersson, R. 2001. Höstbiologi och sångaktivitet hos svarta rödstjärten Phoenicurus ochruros i sydvästra Sverige. Ornis Svecica 11: 135-146.

Anonym. 1981. Fenologi och sträckmönster hos några tätttingar i SV-Skåne. Del 1. Fågelstudier 1: 4-27.

Baker, R.R. 1978. The Evolutionary Ecology of Animal Migration. London.

Baker, R.R. 1993. The function of postfledging exploration: a pilot study of three species of passerines ringed in Britain. Ornis Scand. 24: 71-79.

Bensch, S. \& Lindström, A. 1992. The age of young Willow Warblers Phylloscopus trochilus estimated from different stages of post-juvenile moult. Ornis Svecica 2: 23-28.

Berthold, P. 1983. Uber Jugendentwicklung, Zugunruhe und Zugverhalten des Hausrotschwanzes (Phoenicurus ochruros). J. Orn. 124: 117-131.

Berthold, P. 1985. Vergleichende Untersuchung von Jugendentwicklung und Zugverhalten bei Garten und Hausrotschwanz, Phoenicurus phoenicurus und P. ochruros. J. Orn. 126: 383-392.

Biber, O. 1973. Zur phänologie des Herbstzuges beim Hausrötel Phoenicurus ochruros nach Fangergebnissen auf dem Chasserel (Berner Jura). Orn. Beob. 70: 147-156.

Boddy, M. 1983. Factors influencing timing of autumn dispersal or migration in first-year Dunnocks and Whitethroats. Bird study 30: 39-46.

Cramp, S. 1988. Handbook of the Birds of the Western Palearctic. Vol. 5, Oxford.

Ehrenroth, B. 1976. Vandringsrörelser hos mesar Paridae vid Hammarön, norra Vänern. Vår Fågelvärld 35: 261-278.
Glutz von Blotzheim, U.N. \& Bauer, K.M. 1988. Handbuch der Vögel Mitteleuropas. Band 11. Wiesbaden.

Goodbody, I.M. 1952. The post-fledging dispersal of juvenile titmice. British Birds 45: 279-285.

Greenwood, P.J. \& Harvey, P.H. 1982. The natal and breeding dispersal of birds. Annual Review of Ecology and Systematics 13: 1-21.

Jenni, L. \& Winkler, R. 1994. Moult and Ageing of European Passerines. Academic Press, London.

Johansson, L. 1980. Flyttning inom häckningsområdet hos några tättingar. Verks. vid Kvismare fågelstation 1979: 13-16.

Knutsson, T. 1981. Svarta rödstjärten på Öland. Calidris 10: 151-160.

Lindström, Å, Bensch, S. \& Hasselquist, D. 1985. Autumn migration strategy of young Bluethroats Luscinia svecica. Vår Fågelvärld 44: 197-206.

Lindström, Å. \& Lind, J. 2001. Fuel deposition and speed of early autumn migration of juvenile Bluethroats Luscinia s. svecica leaving their natal area in Swedish Lapland. Ornis Svecica 11: 253-264.

Landmann, A. 1996. Der Hausrotschwanz. Vom Fels zum Wolkenkratzer- Evolutionsbiologie eines Gebirgsvogels. Aula Verlag, Wiesbaden.

Menzel, H. 1983. Der Hausrotschwanz. Die Neue BrehmBücherei 475.

Nesenhöner, H. 1956. Beobachtungen, besonders brutbiologischer Art am Hausrotschwanz. Ber. naturw. Ver. Bielefeld 14: 128-167.

Nielsen, B.P. 1977. Danske musevåges treckforhold og spredning. Dansk Orn. For. Tidskr. 71: 1-9.

Nielsen, B. \& Bensch, S. 1995. Postfledging movements of juvenile Reed Warblers Acrocephalus scirpaceus and Sedge Warblers Acrocephalus schoenobaenus. Ornis Svecica 5: 125-131.

Norman, S.C. 1991. Post-juvenile moult in relation to dispersal and migration in the Chiffchaff Phylloscopus collybita. Ringing \& Migration 12: 80-85.

Otterlind, G. 1954. Flyttning och utbredning. Ett bidrag till kännedomen om den skandinaviska fågelfaunans utbredningsdynamik. Vår Fågelvärld 13: 1-31, 83-113, 147-167, 245-261.

Ottosson, U., Ottvall, R., Elmberg, J., Green, M., Gustafsson, R., Haas, F., Holmqvist, N., Lindström, A., Nilsson, L., Svensson, M., Svensson, S. \& Tjernberg, M. 2012. Fåglarna i Sverige- antal och förekomst. SOF, Halmstad.

Paradis, E, Baillie, S.R, Sutherland, W.J. \& Gregory, R.D. 1998. Pattern of natal and breeding dispersal in birds. Journal of Animal Ecology 67: 518-536.

Raine, A.F, Sowter, D.J, Brown, A.F. \& Sutherland, W.J. 2006. Natal philopatry and local movement pattern of Twite Carduelis flavirostris. Ringing \& Migration 23: 89-94.

Steel, C. \& Pedersen, P.H. 1993. Syngende elvesanger Locustella fluviatilis og heckende svartrödstjert Phoenicurus ochruros i Risör, Aust- Agder. Larus Marinus 22: 22-27.

Stervander, M. 1999. Trastsångarens ungfågelrörelser. Fåglar $i$ Kvismaren 14: 2-6.

Svensson, L. 1992. Identification Guide to European Passerines. Stockholm.

Svensson, S., Svensson, M. \& Tjernberg, M. 1999. Svensk Fågelatlas. Vår Fågelvärld, supplement 31, Stockholm.

Waldenström, J. \& Bengtsson, D. 2001. Robin Erithacus 
rubecula found in juvenile plumage on spring migration. Ornis Svecica 11: 265-266.

Zink, G. 1981. Der Zug europäisher Singvögel, 3. VogelzugVerlag. Möggingen.

\section{Summary}

The Black Redstart Phoenicurus ochruros shows an annual culmination of ringed birds in July-August at Ottenby, Falsterbo and Nidingen bird observatories. These birds are primarily yearlings in juvenile plumage, which have left their birthplaces after fledging (Figure 1). Black Redstart yearlings have a unique moult strategy compared to other European thrushes. Species like Redstart and Robin normally begin the postjuvenile moult in the birth area only a few weeks after fledging (Glutz \& Bauer 1988, Landmann 1996, Waldenström \& Bengtsson 2001). Black Redstarts, however, start the partial body moult when they reach an average age of 87 days (Berthold 1983, 1985, Glutz \& Bauer 1988, Landmann 1996). This allows for dispersal of yearlings during the summer, leading them to new locations where they carry out the partial moult. It is also known that yearlings of Bluethroat can begin their migration before the moult is finished (Lindström et al.1985, Lindström \& Lind 2001).

Dispersal of yearlings among passerines is poorly researched (Goodbody 1952, Baker 1978, 1993, Greenwood \& Harvey 1982, Boddy 1983, Norman 1991, Paradis et al. 1998, Raine et al. 2006). In Sweden, the topic has only been touched upon briefly for Black Redstart (Knutsson 1981, Andersson 1986, 2001). This paper intends to give a more detailed picture of the progress of the yearling dispersal in relation to the autumn migration by utilizing data on ringing, weight and moult. It is especially motivated since these dispersal movements have sometimes been perceived as an early start of autumn migration. However, as has previously been confirmed from Central Europe, the summer records are well separated from those of the autumn migration (Menzel 1983, Glutz \& Bauer 1988). Berthold (1983, 1985) shows that migration restlessness and the main observed migration of yearlings occurs much later in October-December, an average of two weeks after the moult is finished. Together with the increased migration restlessness, an increase in weight of experimental birds has also been noted. Adult birds normally also leave their breeding grounds in October-November, after a pronounced singing period in September-October (Cramp 1988, Glutz \& Bauer 1988, Anders- son 2001). However, some movements before the migration seem to occur also in older males.

The material analyzed here includes ringing, weight and moult data from the bird observatories at Ottenby 1980-2011 and Falsterbo and Nidingen 1980-2012. The period that has been analyzed is 20 June through 30 November. On Nidingen trapping has been continuous over the season, but not standardized. At Ottenby trapping has occurred spontaneously until 25 July, from that date it has been standardized. At Falsterbo ringing has been standardized from 21 July. The classification of the postjuvenile moult has followed the score of different moult stages, from completely juvenile plumage to finished first winter plumage (Table 2, Lindström et al 1985, Bensch \& Lindström 1992). The classification presented in this paper was adapted to the different methods used on the bird observatories. In addition, data has been included from a previous population study with color-ringed individuals in western Sweden (Andersson 1987, 1990, 1995, 2001). They concern movements after the breeding period in 19 of 188 ringed nestlings, 34 of 105 territorial males and 8 of 20 females ringed during the spring. This is to provide a complementary picture of yearling distribution patterns, but also of the movements of the adults before the autumn migration.

The number of ringed Black Redstarts at the bird observatories had two distinct peaks in the autumn season (Figure 2). The total percentage of yearlings was 86,98 and $96 \%$ for the three observatories respectively (Table 1). During the early peak in JulyAugust the proportion of yearlings was slightly higher (92, 100 and $96 \%$ ). At Ottenby and Falsterbo 63 and $67 \%$ of the total autumn catch was ringed during July-August, while the corresponding figure for Nidingen was $89 \%$. In September very few birds were ringed as that month coincides with the main yearling moult period.

More yearlings were observed to have commenced moult during July-August at Ottenby $(96 \%)$ than at Falsterbo (40\%) and Nidingen (14\%) (Table 2). However, if also birds in moult stage 1 , representing essentially juvenile plumage, are added to those in stage 0 the percentages are more equal, 80,81 and $93 \%$ respectively. Birds caught in early autumn were lighter than birds caught in late autumn. The average weight for Ottenby birds captured in June-August was 15.3 grams $(\mathrm{n}=106)$ and in October-November 16.3 grams ( $\mathrm{n}=62, \mathrm{~F}=9.47, \mathrm{P}<0.01)$. At Falsterbo, the corresponding figures were $15.0(\mathrm{n}=106)$ and 15.5 $(\mathrm{n}=45, \mathrm{~F}=12.95, \mathrm{P}<0.001)$. The values of Nidin- 
gen was $15.0(n=122)$ and $16.5(n=10, F=20.83$, $\mathrm{P}<0.001$ ).

Out of 188 color-ringed nestlings in western Sweden, 19 were found during the autumn season. Two were stationary during 29 and 68 days, while seventeen were observed only one day in the same place. These yearlings moved to new sites and were observed up to 7000 meters away. Out of 105 color-ringed, territorial males in western Sweden 34 were observed in the autumn. Nineteen of them stayed only 1-5 days in the same place. Seven of these were later encountered 500-2000 meters away, while further observations are missing. Eight of the 20 females that were color-ringed during the breeding season were observed during the autumn. All were very stationary and no movements were recorded beyond 800 meters. The ringing data from Ottenby, Falsterbo and Nidingen bird observatories confirms that the majority of the ringed Black Redstarts in July-August, consists of yearlings that have not undergone or only begun their postjuvenile moult. This indicates, together with lower weights, that this peak does not represent any actual migration. The behavior of colourringed yearlings in western Sweden also supports the idea that yearlings at this time show a high degree of flexibility and mobility. Even some adult males showed surprisingly large non-migratory movements during the autumn season.

Every autumn, there is a significant influx of unringed individuals in the breeding areas of western Sweden, probably a result of extensive dispersal of yearlings. A small part also consists of adult individuals, as suggested by documented adult male mobility (Andersson 2001). Perhaps the majority of the individuals from this influx in western Swe- den as well as the birds that are trapped at Nidingen and Falsterbo in July-August have their origin in southernmost Sweden or Denmark. This could explain why there are no recoveries at Nidingen of yearlings ringed in Gothenburg c. $40 \mathrm{~km}$ to the north, where in some years up to about $20 \%$ of the yearlings have been ringed (own unpublished data). No yearlings from the breeding areas in central Halland (where an even higher proportion of birds have been ringed), have resulted in any controls at either Nidingen (30 km to the north) or Falsterbo (230 km to the south). A major population increase has occurred during recent decades in Denmark (Svensson et al 1999, www.dofbasen.dk/ ART/). In the same period, the population of western Sweden has undergone a drastic decline (Ottosson et al 2012, own unpublished data). It is also possible that some of the birds ringed at Ottenby could have the origin in southern populations. How far yearlings can move during July-August is still unclear. Zink (1981) and Glutz \& Bauer (1988) indicate several instances where young Belgian and German ringed Black Redstarts dispersed in a northerly direction up to a distance of 290 kilometers. It has also been argued that long dispersal of yearlings can play an important role for future territorial and breeding establishments (Baker 1993). There are several cases when birds that had been ringed as yearlings in western Sweden were recovered in the following spring north of the ringing site. One example comes from southern Norway, where a female was found to breed 250 kilometers north-west of the birthplace (Steel 1993). It is not inconceivable that it had moved there during late summer and went through its first moult. 